\title{
Multiscale Modeling for the Analysis of Grain-Scale Fracture within Aluminum Microstructures
}

\author{
Edward H. Glaessgen* \\ NASA Langley Research Center, Hampton, Virginia, 23681 \\ Dawn R. Phillips ${ }^{\dagger}$ \\ Lockheed Martin Space Operations, Hampton, Virginia, 23681 \\ Vesselin Yamakov \\ National Institute of Aerospace, Hampton, Virginia, 23681 \\ and \\ Erik Saether* \\ NASA Langley Research Center, Hampton, Virginia, 23681
}

\begin{abstract}
Multiscale modeling methods for the analysis of metallic microstructures are discussed. Both molecular dynamics and the finite element method are used to analyze crack propagation and stress distribution in a nanoscale aluminum bicrystal model subjected to hydrostatic loading. Quantitative similarity is observed between the results from the two very different analysis methods. A bilinear traction-displacement relationship that may be embedded into cohesive zone finite elements is extracted from the nanoscale molecular dynamics results.
\end{abstract}

\section{Introduction}

$\mathrm{C}$ lassical fracture mechanics is based on the comparison of computed fracture parameters to their empirically determined critical values. Although this paradigm has been extremely successful for modeling crack growth at structural scales, it does not describe the fundamental processes that govern fracture. Ultimately, the understanding of events and processes that occur at length scales on the order of $10^{-9}$ to $10^{-3} \mathrm{~m}$ is needed to understand crack growth.

Multiscale modeling provides an efficient means of interrogating deformation and fracture of metallic materials from the micro- to the nano-scales. Many multiscale modeling strategies have been explored by mechanists in recent years; a few of them, coupling methods, quasicontinuum methods, equivalent continuum mechanics methods, and modeling with cohesive zone models, are discussed here. Coupling methods involve a priori dividing a problem into spatial regions based on the simulation technique to be used and tying those regions together with interface boundary conditions. For crack problems, a recently advocated approach combines $a b$ initio quantum mechanics at the crack tip, molecular dynamics (MD) near the crack surface, and finite element (FE) continuum mechanics in the surrounding medium ${ }^{1-3}$

Quasicontinuum methods ${ }^{3-5}$ employ a continuum framework with a finite element discretization wherein each element is formed by nodes that coincide with "representative" atoms. ${ }^{5}$ In regions where the behavior of each atom is essential to understanding the problem, all atoms are considered as representative and coincide with nodes. In regions where a coarse mesh is appropriate, a finite element may encompass many atoms within its interior in addition to the atoms that coincide with the nodes. Also, it is assumed that the element constitutive relations are obtained directly from atomistic calculations. The Cauchy-Born hypothesis is used to relate homogeneous

\footnotetext{
* Aerospace Engineer, Computational Structures and Materials Branch, MS 155, AIAA Member.

${ }^{\dagger}$ Aerospace Engineer, MS 155, AIAA Member.

${ }^{\ddagger}$ Staff Scientist, MS 155, AIAA Member.
} 
deformations of the continuum to the superposed lattice to obtain the element strain energy by summing up the contributions of the interatomic potentials of the contained atoms. ${ }^{4,5}$

The equivalent continuum mechanics method ${ }^{3,6}$ is similar to the quasicontinuum methods, but uses the meshless local Petrov-Galerkin (MLPG) method rather than finite elements. In general, meshless methods are developed to overcome some of the disadvantages of the finite element method, such as the need to interpolate discontinuous secondary variables across interelement boundaries and the need for remeshing in large deformation problems.

In multiscale modeling with cohesive zone models (CZM) ${ }^{7-9}$ the CZM can be inserted within finite element models of microstructures, either within grains to model transgranular fracture or along grain boundaries to model intergranular fracture. Typically, the properties of the CZM are determined empirically or heuristically. However, an ideal CZM-based multiscale modeling strategy would use MD results as input to the CZM. Thus, the CZM may be used as a means to combine these two methods to bridge length and time scales and to provide the basis of a modeling approach that spans from nanometers to millimeters.

In this paper, multiscale modeling is explored to study intergranular fracture in aluminum, with molecular dynamics and the finite element method (FEM) as the building blocks. First, MD and the FEM are discussed individually to highlight their potential in the proposed multiscale modeling strategy. Previous attempts to combine the two are also mentioned. Then, MD and FE simulations on a bicrystal model are performed to extract the constitutive behavior of the grain boundaries. Finally, a method of recasting this information in the form of cohesive zone models is discussed.

\section{Background on Elements of a Multiscale Modeling Strategy}

\section{A. Molecular Dynamics}

To effectively model polycrystalline materials at the atomic scale, large numbers of atoms are needed. Approximations describing the potential energy of the interactions between neighboring atoms have been developed in the form of empirical and semi-empirical potentials. ${ }^{10}$ These potentials are defined for atom-pair and many-body force interactions. An MD simulation is made tractable for large systems of atoms by treating each atom as a point mass, summing forces due to interactions with surrounding atoms, and using Newton's Second Law over a succession of time steps to obtain trajectories specifying atom positions and velocities.

In the case of metals, metallic bonding is formed through delocalized electrons that are effectively shared between all atoms in the lattice. Thus, simple atom-pair potentials cannot be used to account for all of the mutual interactions between the electron clouds surrounding the atoms. Modifications, such as cluster potentials, pair functionals, and cluster functionals, have been made to pair potentials to develop general classes of interatomic potentials. The embedded-atom method (EAM), which uses a pair functional and treats each atom as being embedded in a field of electrons created by the surrounding atoms, has been successful in simulating the atomic bonding in metals. ${ }^{11}$

Considerable attention has been given to the atomic-level dynamic instabilities that occur during fracture. In homogeneous media, these instabilities may result in crack branching and roughness at the crack surface dramatically increasing the crack area and the energy dissipation at the crack tip. ${ }^{12}$ In ductile polycrystals, such as face centered cubic (FCC) metals, the transgranular crack propagation taking place through the grains experiences a "dynamic brittle-to-ductile transition" as a result of the dynamic instability. ${ }^{12}$ The transition is associated with blunting of an initially brittle crack in an explosion of dislocations at the crack tip ${ }^{12}$ together with crack branching along the available slip planes and surface roughening. ${ }^{13}$ In intergranular fracture, in which cracks propagate along the grain boundary (GB) interface, the crack tip is channeled by the GB layer. This constrained condition may affect the crack dynamics and instability in the crack motion and yield significantly different behavior compared with transgranular fracture.

\section{B. Finite Element Modeling with Cohesive Zone Models}

Cohesive zone models approximate an interfacial traction-displacement relationship using bulk material properties and can model the appearance of fracture surfaces in a continuum. ${ }^{14}$ Although the normal and shear components of the traction and displacement may be considered separately, as in reference \& a more realistic approach may be to consider that the components do not act independently of each other. In the Tvergaard and Hutchinson $^{7}$ coupled cohesive zone model (CCZM), the normal and shear components of the traction and displacement are combined into single measures, $\tau$ and $\lambda$, respectively, so that the responses are coupled. ${ }^{9}$ The CCZM given in reference 9 defines a traction potential, ${ }^{15}$ 


$$
\Phi\left(\delta_{n}, \delta_{t}\right)=\delta_{n}^{c} \int_{\lambda} \tau\left(\lambda^{\prime}\right) d \lambda^{\prime}
$$

where $\lambda$ is a non-dimensional measure of the relative opening and sliding displacements $\left(\delta_{n}\right.$ and $\left.\delta_{t}\right)$ as defined by

$$
\lambda=\left[\left(\frac{\delta_{n}}{\delta_{n}^{c}}\right)^{2}+\left(\frac{\delta_{t}}{\delta_{t}^{c}}\right)^{2}\right]^{1 / 2}
$$

$\delta_{n}{ }^{c}$ and $\delta_{t}^{c}$ are the critical values for the opening and sliding modes, respectively, and a fracture surface is assumed to have formed once $\lambda$ reaches a value of unity. The use of cohesive zone models in finite elements permits highly discontinuous displacement behavior to be exhibited in a single discretized model without remeshing while using an incremental piecewise linear solution algorithm. The displacement discontinuity may be defined in three separate regimes that depend on the relative CZM element nodal displacements. First, the CZM element functions as an elastic constraint to enforce node coincidence at the GB between connected continuum elements. Secondly, during strain softening of the opening of the CZM element, the effective stiffness is reduced as a function of the relative displacements of initially coincident nodes. Finally, after the boundary has opened and the effective stiffness becomes zero, further increments may cause local load redistribution resulting in separated surfaces coming back into contact. In this regime, the CZM element functions as a contact element to prevent interpenetration.

As a consequence of the unique numerical features of the CZM element, a number of issues related to convergence exist that must be addressed prior to performing a successful analysis. One issue involves the magnitude of the initial "penalty stiffness" that is used to enforce the elastic constraint. This quantity is problemspecific and can lead to convergence difficulties if it causes the global stiffness matrix to become ill-conditioned. ${ }^{16}$ Also, if interpenetration is detected after a boundary has opened, the abrupt replacement of zero stiffness terms between surface elements with penalty stiffness values can cause divergence of the solution. Another issue pertains to the integration scheme used to compute the stiffness coefficients for the CZM element. In some instances, a Gaussian quadrature scheme can contribute to poor convergence compared to a Newton-Cotes scheme. ${ }^{17}$ Solution convergence is also dependent on the size of the CZM elements compared to the extent of the process zone surrounding the fracture front. ${ }^{18}$ An additional issue pertains to the possible extensive use of CZM elements within a model. If no a priori knowledge of crack paths is available, CZM elements may be placed between all continuum elements such that the crack path will be automatically determined by the predicted stress fields. However, if the initial or "penalty" stiffness is set to a small value, this can introduce an artificial flexibility into the model that can alter load transfer and displacements.

\section{Combining Molecular Dynamics and Finite Elements}

Attempts to extract relevant parameters for the decohesion law of a CZM element from atomistic (moleculardynamics or molecular-static) simulations have been made in the last few years. ${ }^{19}$ The approach in reference 19 is based on simulating the debonding of a flat interface with no initial flaw under a constant tensile strain rate perpendicular to the interface. In these models, the predicted mechanism for interface decohesion is not that of crack propagation, but rather of atom adhesion at the interface. Thus, the simulations reproduce the process of adhesion rather than that of mechanical fracture at the GB. Raynolds, et al. ${ }^{20}$ use a similar setup for studying, by first principles calculations, the conditions and the mechanisms for initial void formation and crack initiation in adhesion curves of an NiAl-Cr interface. However, the resulting adhesion curves cannot be used directly to derive the constitutive decohesion laws for CZM elements because the boundary conditions at which a typical CZM element operates in a large scale FEM model are very different from the ones used in the MD simulations.

\section{Elements of a Multiscale Modeling Strategy}

The work presented in this paper is a potential step toward an integrated multiscale modeling strategy for understanding the connection between macroscopic fracture and the details of the underlying atomistic failure mechanisms. The range of relevant length scales span from the atomistic $\left(10^{-9} \mathrm{~m}\right)$ as shown in Fig. 1(a) to the macroscopic $\left(>10^{-3} \mathrm{~m}\right)$ as shown in Fig. 1(f). Inherent to multiscale modeling across length scales is a trade-off between mechanistic detail and numerical efficiency. The mapping of the relevant physics between length scales necessarily involves a homogenization procedure to obtain an aggregate response over a representative volume of 


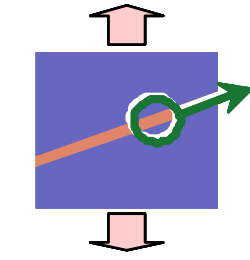

Grain Decohesion and Dislocation Motion

(a) Atomistic simulations

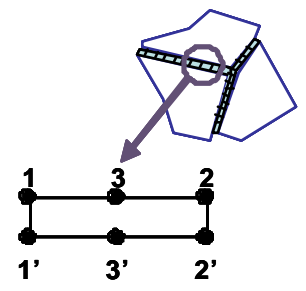

(c) Embedding atomistics in finite element models
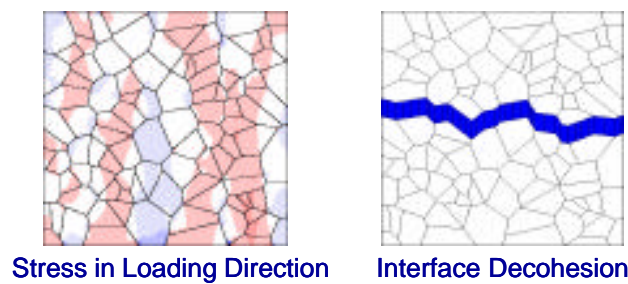

(e) Response of metallic microstructures under mechanical loads

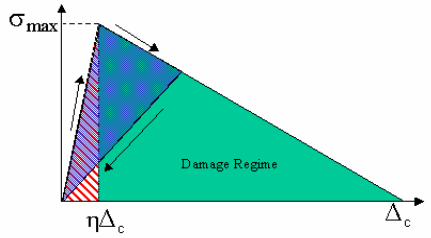

(b) Continuum interpretation of atomistic results

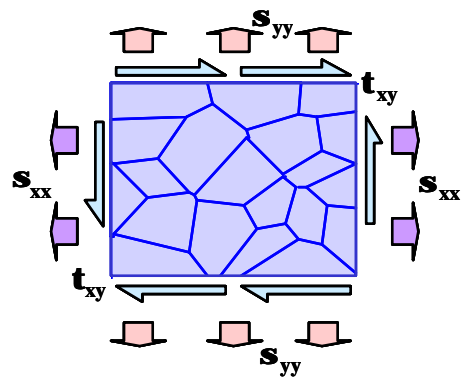

(d) Idealization of metallic microstructures

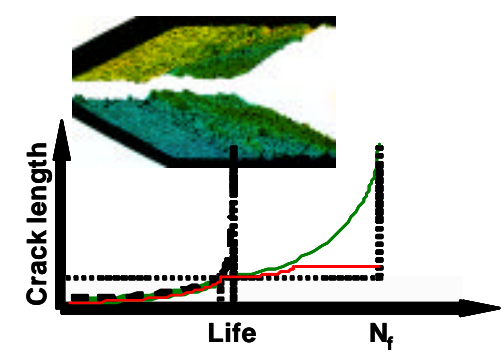

(f) Macroscopic measures of crack growth

Figure 1. Multiscale modeling with cohesive zone models.

material. In general, this homogenization is performed at each increase in length scale and aims to preserve pertinent measures of fracture while reducing the number of independent variables. Within this modeling paradigm, various computational methodologies must be integrated to provide an effective homogenization. The particular homogenization used herein involves modeling the material behavior at the atomistic level using MD simulations and recasting the discrete interactions as local continuum field quantities through a cohesive zone modeling approach.

The specific problem of intergranular fracture is considered wherein the MD simulations of a bicrystal (Fig. 1(a)) are used to obtain an atomistic response of crack propagation under applied loads. This response is then recast to obtain an averaged continuum traction-displacement relationship (Fig. 1(b)) to represent the cohesive interaction along a characteristic length of the GB (Fig. 1(c)). For the present stage of ongoing research, the resulting CZM is utilized as a bridge between atomistic and local continuum field modeling (Fig. 1(d,e)). The determination of the dependence of the derived constitutive relation on length scale within a continuum representation is an ongoing research objective.

\section{A. Molecular Dynamics and Finite Element Analyses on Elastically Equivalent Systems}

A simple bicrystal model with a single grain boundary is used to study the behavior of a particular system as predicted by molecular dynamics and the finite element method. If these two fundamentally different analysis techniques can be shown to yield consistent results for this problem, then there exists a solid basis for the proposed multiscale modeling strategy.

The MD simulation set-up used in the current analysis represents a thin bicrystal strip with periodic boundary conditions in all three directions. The atomic microstructure of the system is presented in Fig. 2. The normal to the 


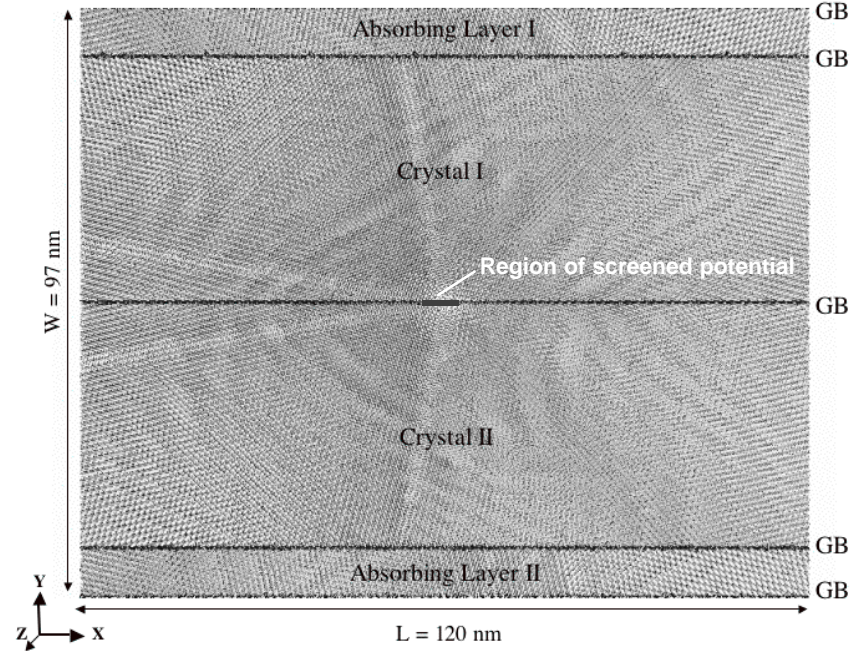

Figure 2. Initial atomistic snapshot of the MD system before crack propagation. (more than four times larger than the range of the interatomic potential ${ }^{23}$ ) to prevent interference of the atoms with their periodic images along the $z$-direction, thus preserving the local three-dimensional physics in the system. The configuration allows the system size in the $x$-and $y$-directions to extend up to 120 and 97 nm, respectively, while limiting the number of simulated atoms to 1,994,000, thus allowing the simulation to be carried out on a modest Beowulf cluster. In addition, the $\langle 1 \quad 10>$ texture makes two of the four $\{111\}$ slip planes in the FCC lattice available for dislocation glide and cross-slip events, as in a fully $3 \mathrm{D}$ space. $^{25}$

The two narrower layers on both sides of the bicrystalline system (Absorbing Layer I and II in Fig. 2) serve as shock-wave absorbers, ${ }^{26}$ where a damping friction coefficient is applied to the atoms to absorb the phonon waves ${ }^{26}$ expected to be generated from the crack tips. The additional GBs formed between these layers and Crystals I and II act as absorbers for the dislocations that may be emitted from the crack tips during propagation. The inclusion of these shock-wave and dislocation absorbers suppresses the creation of periodic images of all the disturbances emitted from the crack tips, thus avoiding the unrealistic influence these disturbances would otherwise have on the crack propagation.

The simulated strip is prestrained by applying a constant external hydrostatic load $\left(\sigma_{x x}=\sigma_{y y}=\sigma_{z z}=\sigma\right)$ at a constant temperature of $100 \mathrm{~K}$. The temperature $100 \mathrm{~K}$ is chosen to suppress grain boundary and surface diffusion. After reaching mechanical equilibrium, the system size is fixed. The hydrostatic loading helps to eliminate plasticity effects not related to the crack, such as spontaneous dislocation nucleation from the GB, ${ }^{25}$ which could otherwise dominate the deformation in this (essentially) two-dimensional configuration. The crack is initiated by screening the interatomic potential between atoms on both sides of the Crystal I-Crystal II GB plane (Fig. 2) along a region of $5.7 \mathrm{~nm}$ along the middle of the GB. From this initial crack, the GB opens, and the crack starts to propagate in both directions along the GB interface. Figure 3 shows MD snapshots of cracks that propagate in a plate prestessed at initial hydrostatic loads of $\sigma=3.5,3.75,4.0$, and $4.25 \mathrm{GPa}$. Common neighbor analysis $(\mathrm{CNA})^{27}$ is used to identify atoms in different crystallographic states: FCC, HCP, and non-crystalline atoms (gray, red, and blue in Fig. 3, respectively). Atoms with more than 1/3 of their nearest neighbors missing are identified as surface atoms (green in Fig. 3). In each of the cases presented, the crack propagation is not symmetric in the $+x$ and $-x$ directions along the GB (as defined in Fig. 2). The propagation in the $-x$ direction produces two twin patterns (1) in Fig. 3) that grow almost symmetrically in the two joined crystals and retard the $-x$ direction propagation of the crack. Also observed in the snapshots are stacking faults (2) in Fig. 3) and partial dislocations (3) in Fig. 3) that originate near the $-x$ direction tip of the crack. By contrast, the propagation in the $+x$ direction is more brittle, accompanied by only a few perfect dislocation emissions (4) in Fig. 3). As a result, the crack propagates much further along the GB in the $+x$ direction.

The corresponding FE configuration is presented in Fig. 4. The dimensions of the system are scaled to recover the proportions of the MD system. The model contains a built-in lenticular slit of varying relative length, $0.05=l / W$ $=0.91$, to study the evolution of the system at different stages of crack growth. Although the lenticular slit is not a crack in the true continuum mechanics sense, it is an analog to the MD configuration and hence is used to represent a crack-like discontinuity (hereafter referred to as a crack for simplicity). To avoid stress singularities in the FE 

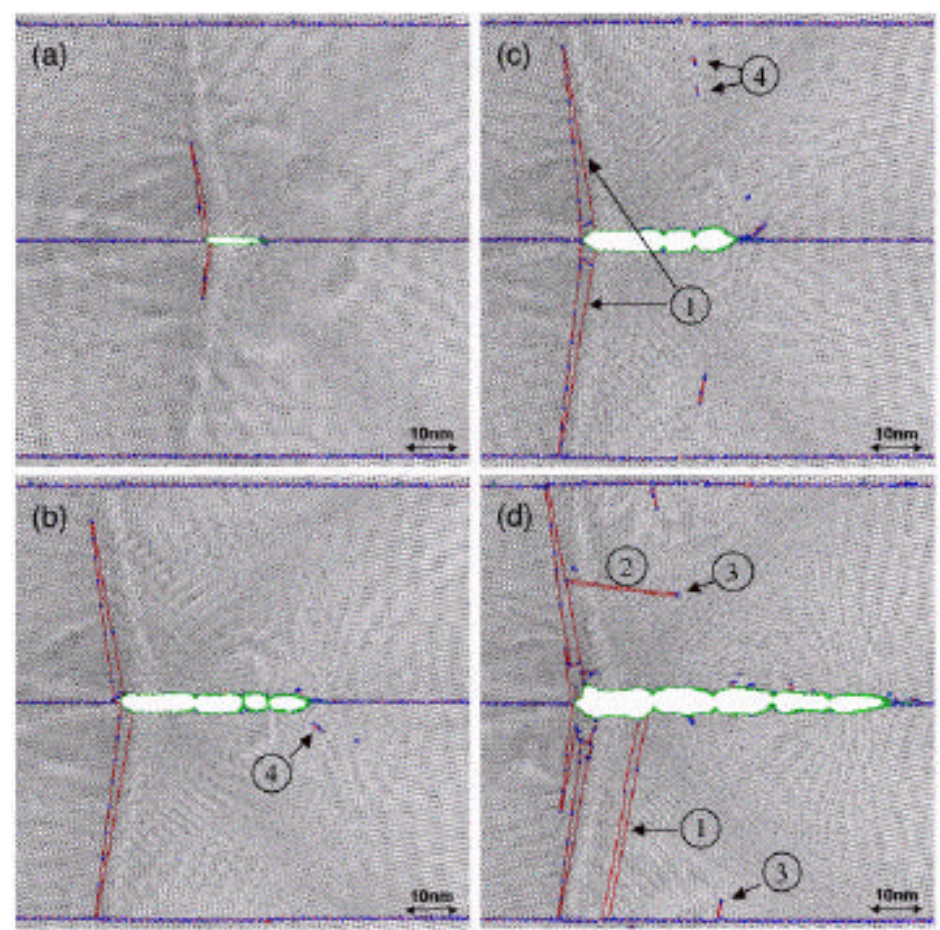

Figure 3. MD snapshots of cracks propagating in the MD system shown in Fig. 2 prestressed at initial hydrostatic loads of $\sigma=$ (a) 3.5, (b) 3.75, (c) 4.0, and (d) 4.25 GPa. system, the crack tips have a finite, but very small, initial radius that corresponds to an initial crack opening of approximately $1 \mathrm{~nm}$, i.e., slightly larger than the range of the interatomic forces $(0.67 \mathrm{~nm})$. There is no elastic equivalent for the $\mathrm{GBs}$ in the $\mathrm{FE}$ system. It is assumed that the somewhat different elastic response of the GB layers does not alter significantly the crack behavior because of the relatively small volume ratio of the GB volume in the system. The matrix is assumed to be anisotropic linear elastic, with constants assigned according to the MD interatomic potential. Generalized plane strain is used, and the calculations are carried out under displacement control in the $x$ - and $y$ directions. No dynamic or non-linear effects are incorporated, and the results are relevant for infinitely slow crack propagation under small strain.

Plastic processes (twinning and dislocation emission $^{25}$ ) at the crack tips have a pronounced effect on the stress distribution around the growing crack. This effect is revealed by comparing the stress distributions obtained from the MD and the FE simulations of the two elastically equivalent models. The MD simulation necessarily incorporates all the plastic processes together with the elastic response of the bicrystal, while the FE model can be used to predict the elastic response separate from the plastic components of the solution.

The MD simulation necessarily incorporates all the plastic processes together with the elastic response of the bicrystal, while the FE model can be used to predict the elastic response separate from the plastic components of the solution. The MD stress distribution is determined after the atomistic system reaches elasto-plastic equilibrium and the crack stops growing, while the FE stress distribution is determined for a static solution when the system is in elastic equilibrium. Two-dimensional $(x-y)$ stress-maps for the MD results are created by averaging the local virial stress over a chosen volume of $6 \times 6 \times 10 / \sqrt{ } 2$ lattice parameters in the $x$-, $y$-, and $z$-directions, respectively $(2.43 \times 2.43$

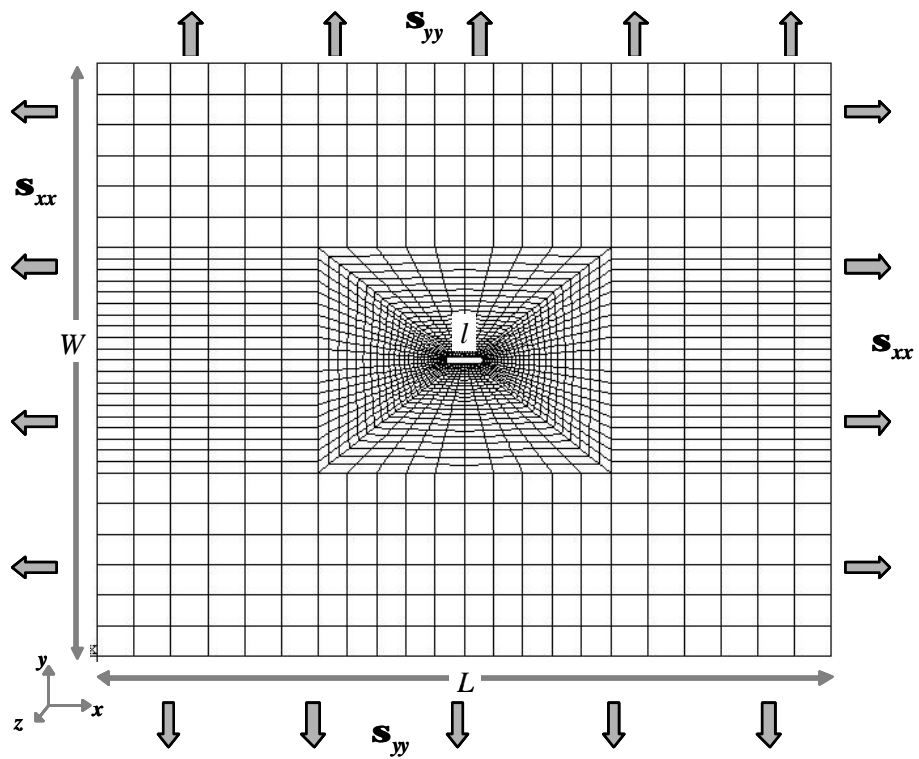

Figure 4. Finite element mesh of model with built-in lenticular crack. $\mathrm{x} 2.86 \mathrm{~nm}^{3}$ volume including 864 atoms) and over 16 ps of simulation time. It should be noted that the virial theorem, which is the basis for calculating the virial stress, provides the oldest and most frequently used expression for relating forces and motion within an atomic system to a continuum stress. In the limit of time and ensemble averages, the virial stress coincides with the Cauchy stress used in continuum mechanics. ${ }^{28}$

Figure 5 presents a comparison between the stress distributions obtained from the FE (Fig. 5(a-c)) and MD (Fig. 5(d-f)) simulations for the case of the small prestress of $3.5 \mathrm{GPa}$, which produces a $10 \mathrm{~nm}$ crack in the MD simulation at equilibrium (seen also in Fig. $3(a))$. The size of the crack is sufficiently small compared to the system size, and the elastic stress field calculated by the corresponding FE simulation does not experience edge effects from the boundary 

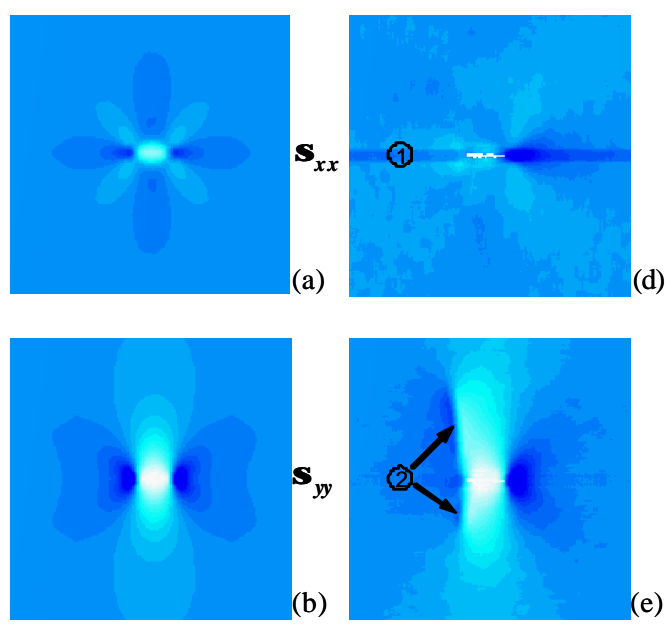

(e)

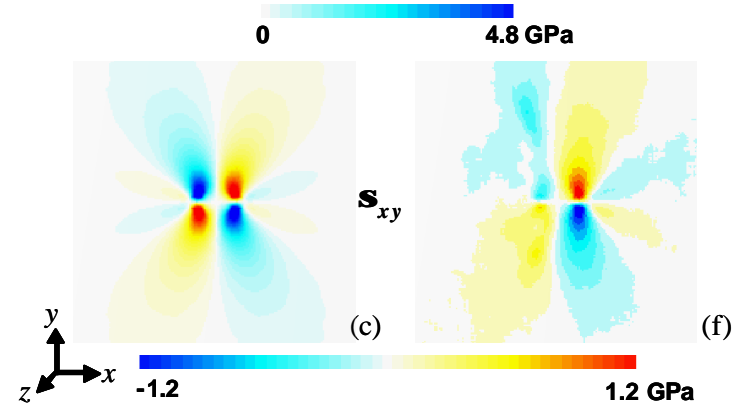

Figure 5. Stress contours from the FEM model and the corresponding stress maps from the MD model. conditions and thus behaves as an infinite plate. The MD stress field captures the GB effect on the stress (1) in Fig. 5(d)). The MD results for stress distribution shown in Fig. 5 (d-f) for the $+x$ crack tip are in very good quantitative agreement with the FE results shown in Fig. 5(a-c). This similarity implies that the crack propagation in the MD system in the $+x$ direction is almost brittle with essentially no plasticity. For the stress distribution for the $-x$ crack tip, there is a large difference between the MD results shown in Fig. 5(d-f) and the FE results shown in Fig. 5(a-c). This difference shows that the twinning observed at the $-x$ crack tip in the MD system significantly alters the stress; the $\sigma_{x x}$ and $\sigma_{x y}$ stress components are largely relieved, while the $\sigma_{y y}$ stress (2) in Fig. 5(e)) is redistributed away from the crack tip along the twin boundaries (see Fig. 3(a)). This redistribution explains the very slow crack propagation in the $-x$ direction of the MD model. As expected, the FE stress distribution is symmetric for the two crack tips because they are elastically equivalent in the FE model (Fig. 5(a-c)).

For a crack tip propagating in an essentially elastic fashion (i.e., the $+x$ crack tip in Fig. 5), consistent results are obtained by the MD and FE simulations, indicating that there is a solid basis for the proposed multiscale modeling strategy for brittle fracture modes. The extraction of a traction-displacement relationship for the $+x$ crack tip from MD results to be used in larger-scale FE calculations is discussed next .

\section{B. Defining a Traction-Displacement Relationship from MD}

Grain-scale simulations that use CZM to study fracture (such as those presented in reference 9) typically use heuristically derived relationships and input values to define the CZM. In such an approach, these values tend to be only gross estimates of parameters such as GB yield stress; however, a cohesive formulation can be part of an effective physics-based approach if constitutive parameters are used that optimize the similitude between atomistic simulation and continuum finite element results. The model can be developed from results of the MD analyses allowing the physical insight of MD to be embedded in the more computationally efficient FE models.

The traction-displacement function of a CZM describes how the traction $\tau$ developed at the crack surface depends on the crack opening $\lambda$ (see Fig. 6). In general, traction-displacement functions must be defined for both normal and tangential components of traction. Here, the special case of normal opening (mode I) under hydrostatic load is considered. The tensile component of the normal stress near the debonding GB interface from the MD simulation, $\sigma_{y y}^{s}$, can be used as an equivalent of the normal traction $\tau_{n}$ in the CZM. To obtain the mode I traction-displacement relationship, the MD simulation box is divided into thin slices of length $\Delta$, as shown in Fig. 7. A set of test volumes (the dotted areas in Fig. 7) of dimensions $\Delta \times 2 \Delta \times \psi$ centered at the debonding interface are defined. For each test volume along the GB

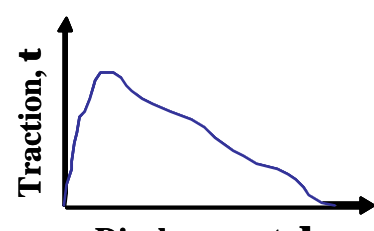
Displacement, $\lambda$

Figure 6. A general traction-displacement relationship. interface, the normal opening of the crack $\delta_{n}$ as a function of $x$ is estimated, and the local normal stress $\sigma_{y y}$ is averaged to obtain $\sigma_{y y}^{s}$ as a function of $x$. Thus, calculating $\sigma_{y y}^{s}(x)$ and $\delta_{n}(x)$ for each test volume at position $x$ along the debonding interface, one an develop the functional relationship $\sigma_{y y}^{s}\left(\delta_{n}\right)$, which represents the tractiondisplacement curve of a CZM volume element (CZVE) of size $\Delta \times 2 \Delta \mathrm{x} \psi$. By definition, $\Delta$ must be small enough so that the stress variation over the CZVE is much smaller than the stress itself. In the present simulation, $\Delta \cong 1.9$ $\mathrm{nm}$, which, with a thickness in the $z$-direction of $2.86 \mathrm{~nm}$, gives a volume of $2 \times 1.9^{2} \times 2.86=20.7 \mathrm{~nm}^{3}$ and contains about 1250 atoms that contribute to the stress-displacement response of one CZVE. There are 63 CZVE along the $120 \mathrm{~nm}$ long GB interface in the system (Fig. 2). During the simulation, the $\sigma_{y y}^{s}\left(\delta_{n}\right)$ state is scanned every 4 ps for a period of $200 \mathrm{ps}$, resulting in 3000 values from which to determine the $\sigma_{y y}^{s}\left(\delta_{n}\right)$ dependence for each simulation run. 


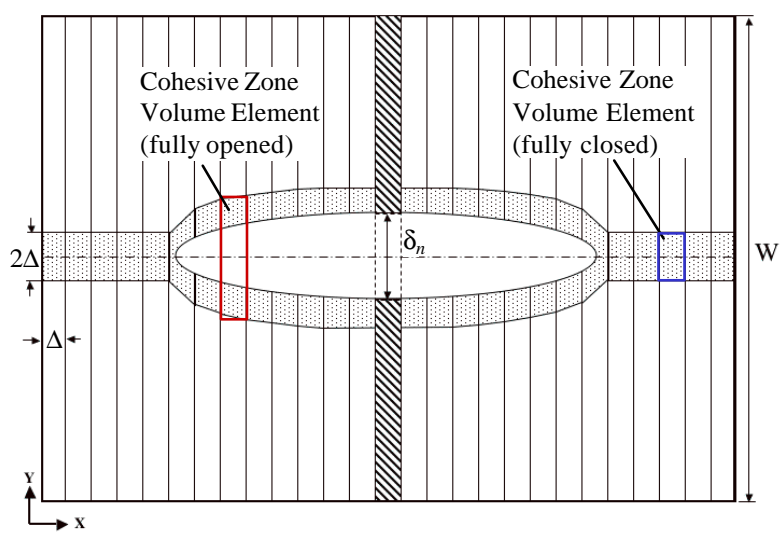

Figure 7. Slicing of the system volume in the MD simulation for extracting the parameters for the cohesive zone volume elements (not to scale).

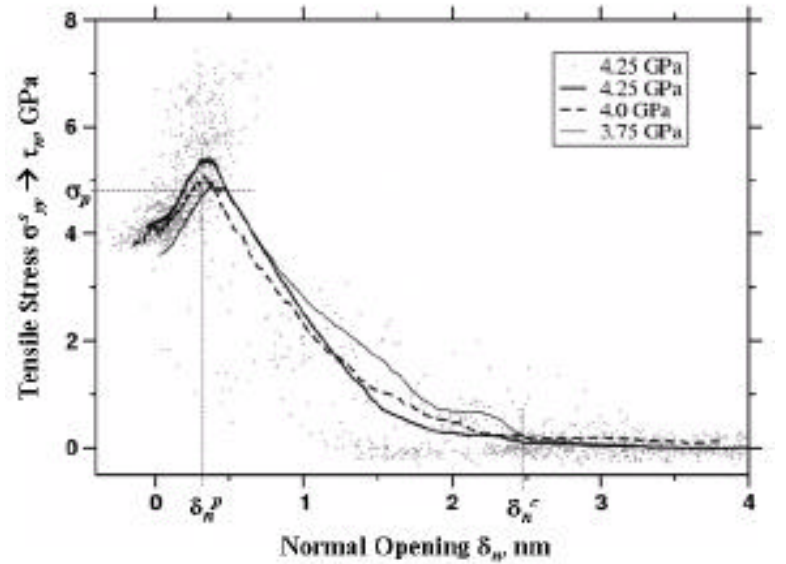

Figure 8. Surface stress vs. crack opening curves, $\sigma_{y y}^{s}\left(\delta_{n}\right)$, characterizing the propagation of the cleavage tip in the $+x$ direction for three prestrain loads.

Fifteen hundred $\sigma_{y y}^{s}\left(\delta_{n}\right)$ values are taken from the CZVE on the right half of the model for the case of $4.25 \mathrm{GPa}$ prestress and are presented as points in Fig. 8. The spread of the data points shows that at this small length scale of $2 \mathrm{~nm}$, there is a large dispersion around the mean traction-displacement curve. These points represent the behavior of the crack as it propagates in the $+x$ direction (see Fig. 5). The $+x$ direction tip propagates more than $50 \mathrm{~nm}$ (Fig. 3), causing $25 \mathrm{CZVE}$ to experience a complete transition from a fully closed to a fully opened state. The $\sigma_{y y}^{s}\left(\delta_{n}\right)$ curve for the $4.25 \mathrm{GPa}$ prestress is extracted by taking a moving average of the $\sigma_{y y}^{s}\left(\delta_{n}\right)$ values over $0.0 \leq \delta_{n} \leq 4.0$ $\mathrm{nm}$. This process is repeated for the cases of $3.75 \mathrm{GPa}$ and $4.0 \mathrm{GPa}$ prestress, and the resulting $\sigma_{y y}^{s}\left(\delta_{n}\right)$ curves are also shown in Fig. 8 (note that the corresponding $\sigma_{y y}^{s}\left(\delta_{n}\right)$ values are omitted from the figure). The curves in Fig. 8 show a bilinear type of constitutive relation for the CZVE similar to the CZM suggested by Camacho and Oritz ${ }^{8}$ and show that for the case of crack propagation in the $+x$ direction for a $\Sigma 99$ STGB in FCC metals, this bilinear model is a good assumption. In addition, Fig. 8 shows that $\sigma_{y y}^{s}\left(\delta_{n}\right)$ is a weak function of prestress of the system over the range $3.75 \mathrm{GPa} \leq \sigma \leq 4.25 \mathrm{GPa}$ and is approximately $\sigma_{p} \cong 5.0 \mathrm{GPa}$ at $\delta_{n}^{p} \cong 0.4 \mathrm{~nm}$. The full opening (debonding) of the interface happens at $\delta_{n}^{c} \cong 2.5 \mathrm{~nm}$, when $\sigma_{y y}^{s}$ becomes zero.

\section{Concluding Remarks}

The present multiscale modeling strategy for the analysis of aluminum microstructures is based on embedding atomic-scale information into finite element simulations by means of cohesive zone models. Analysis of crack propagation in a simple bicrystal model by means of both molecular dynamics and elastically equivalent finite element analysis reveals a quantitative similitude in stress distribution for cracks propagating along a grain boundary. By examining the tensile component of the stress and the crack opening from the atomistic results, a bilinear traction-displacement relationship is developed that incorporates the behavior of approximately 1250 atoms into one equivalent cohesive zone volume element.

\section{Acknowledgments}

V. Yamakov and D. R. Phillips were sponsored through cooperative agreement NCC-1-02043 with the National Institute of Aerospace and contract NAS1-00135 with Lockheed Martin Space Operations, respectively.

\section{References}

${ }^{1}$ Broughton, J. Q., Abraham, F. F., Bernstein, N., and Kaxiras, E., "Concurrent Coupling of Length Scales: Methodology and Application," Physical Review B, Vol. 60, No. 4, 1999, pp. 2391-2403.

2 Abraham, F. F., Bernstein, N., Broughton, J. Q., and Hess, D., "Dynamic Fracture of Silicon: Concurrent Simulation of Quantum Electrons, Classical Atoms, and the Continuum Solid," Materials Research Society Bulletin, Vol. 25, 2000 , pp. $27-32$.

${ }^{3}$ Shen, S. and Atluri, S. N., "Computational Nano-Mechanics and Multi-Scale Simulation," CMC: Computers, Materials, \& Continua, Vol. 1, No. 1, 2004, pp. 59-90.

4 Tadmor, E. B., Ortiz, M., and Phillips, R., "Quasicontinuum Analysis of Defects in Solids," Philosophical Magazine A, Vol. 73, 1996, pp. 1529-1563. 
5 Miller, R. E. and Tadmor, E. B., “The Quasicontinuum Method: Overview, Applications, and Current Direction,” J. Computer-Aided Materials Design, Vol. 9, 2002, pp. 203-239.

${ }^{6}$ Shen, S. and Atluri, S. N., "Multiscale Simulation Based on the Meshless Local Petrov-Galerkin (MLPG) Method," CMES: Computer Modeling in Engineering \& Sciences, Vol. 5, No. 3, 2004, pp. 235-255.

7 Tvergaard, V. and Hutchinson, J.W., "The Relation Between Crack Growth Resistance and Fracture Process Parameters in Elastic-Plastic Solids," Journal of the Mechanics and Physics of Solids, Vol. 40, 1992, pp. 1377-1397.

${ }^{8}$ Camacho, G.T., Ortiz, M., "Computational Modeling of Impact Damage in Brittle Materials," Int. J. Solids Struct., Vol. 33 , 1996, pp. 2899-2938.

9 Iesulauro, E., Ingraffea, A. R., Arwade, S. R., and Wawrzynek, P. A., "Simulation of Grain Boundary Decohesion and Crack Propagation in Aluminum Microstructure Models," Fatigue and Fracture Mechanics: 33 ${ }^{\text {rd }}$ Volume, ASTM STP 1417, 2001.

${ }^{10}$ Raabe, D., Computational Materials Science: The Simulation of Materials Microstructures and Properties, Wiley-VCH, Weinheim, 1998.

${ }^{11}$ Daw, M.S., Foiles, S.M., and Baskes, M.I., “The Embedded-Atom Method: A Review of Theory and Applications,” Mat. Sci. Reports, Vol. 9, 1992, pp. 251-310.

${ }^{12}$ Abraham, F. F., “The Atomic Dynamics of Fracture," J. Mech. Phys. Sol., Vol. 49, 2001, pp. 2095-2111.

${ }^{13}$ Farkas, D., "Bulk and Intergranular Fracture Behaviour of NiAl," Phil. Mag. A, Vol. 80, No. 6, 2000, pp. 1425-1444.

${ }^{14}$ Klein, P. and Gao, H., "Crack Nucleation and Growth as Strain Localization in a Virtual-Bond Continuum," Engineering Fracture Mechanics, Vol. 61, 1998, pp. 21-48.

${ }^{15}$ Tvergaard, V. and Hutchinson, J.W., "The Influence of Plasticity on Mixed-Mode Interface Toughness," Journal of the Mechanics and Physics of Solids, Vol. 41, 1993, pp. 1119-1135.

${ }^{16}$ Schellekens, J. C. J., de Borst, R., "On the Numerical Integration of Interface Elements,” Int. J. Numer. Meth. Engng., Vol. 36, 1992, pp. 43-66.

${ }^{17}$ Camanho, P.P. and Davila, C. G., "Mixed-Mode Decohesion Finite Elements for the Simulation of Delamination in Composite Materials," NASA/TM-2002-211737.

${ }^{18}$ Turon, A., Davila, C. G., "An Engineering Solution for using Coarse Meshes in the Simulation of Delamination using Cohesive Zone Models," NASA TM, 2005 (to be published).

${ }^{19}$ Gall, K., Horstemeyer, M.F., Van Schilfgaarde, M., Baskes, M.I., "Atomistic Simulations on the Tensile Debonding of an Aluminum-Silicon Interface,” J. Mech. Phys. Solids, Vol. 48, 2000, pp. 2183-2212.

${ }^{20}$ Raynolds, J.E., Smith, J.R., Zhao, G.-L., Srolovitz, D.J., “Adhesion in NiAl-Cr from First Principles,” Phys. Rev. B, Vol. 53, 1996, pp. 13883-13890.

${ }^{21}$ Dahmen, U., Hetherington, J. D., O'Keefe, M. A., Westmacott, K. H., Mills, M. J., Daw, M. S., and Vitek, V., “Atomic Structure of a $\Sigma 99$ Grain Boundary in Al: A Comparison Between Atomic-Resolution Observation and Pair-Potential and Embedded-Atom Simulations," Phil. Mag. Lettrs. Vol. 62, 1990, pp. 327-335.

${ }^{22}$ Weertman, J. and Weertman, J. R., Elementary Dislocation Theory, Oxford University Press, New York, 1992.

${ }^{23}$ Mishin, Y., Farkas, D., Mehl, M.J., and Papaconstantopoulos, D.A., "Interatomic Potentials for Monoatomic Metals from Experimental Data and ab initio Calculations," Phys. Rev. B., Vol. 59, 1999, pp. 3393-3407.

${ }^{24}$ Wolf, D., "Structure-Energy Correlation for Grain Boundaries in F.C.C. Metals - III. Symmetrical Tilt Boundaries," Acta Metal., Vol. 38, 1990, pp. 781-790.

${ }^{25}$ Yamakov, V., Wolf, D., Phillpot, S.R., Mukherjee, A.K, and Gleiter, H., "Dislocation Processes in the Deformation of Nanocrystalline Al by Molecular-Dynamics Simulation," Nature Materials, Vol. 1, 2002, pp.45-48.

${ }^{26}$ Gumbsch, P., Zhou, S. J., Holian, B. L., "Molecular Dynamics Investigation of Dynamic Crack Stability," Phys. Rev. B, Vol. 55, 1997, pp. 3445-3455.

${ }^{27}$ Honeycutt, J. D., Andersen, H. C., "Molecular Dynamics Study of Melting and Freezing of Small Lennard-Jones Clusters," J. Phys. Chem., Vol. 91, 1987, pp. 4950-4963.

${ }^{28}$ Zimmerman, J. A., Jones, R. E., Klein, P. A., Bammann, D. J., Webb, E. B. III, and Hoyt, J. J., “Continuum Definitions for Stress in Atomistic Simulation," SAND Report, SAND2002-8608. 